\title{
Geometry in a Manifold with Projective Structure`
}

\author{
J. Ehlers \\ Max-Planck Institut für Physik und Astrophysik, München, Germany
}

A. Schild

The University of Texas, Austin, Texas, USA

Received February 5, 1973

\begin{abstract}
Parallel transport of line elements, surface elements etc. along geodesics and more general curves in a projectively connected manifold is investigated analytically and in terms of geometrical constructions. Projective curvature is characterized geometrically by a projective analogue of the geodesic deviation equation and by a geometrical construction. The results are interpreted physically as statements about free fall world lines in space-time.
\end{abstract}

\section{Introduction; Geometry of Free Fall and Light Propagation}

The theory of special relativity, developed by Lorentz, Poincaré, and Einstein in the years 1902-1905 and perfected by Minkowski in 1908 has as its basic ingredient the properties of light propagation. It is these properties which suggest the ideas of an absolute limiting speed, the behavior of time (no absolute simultaneity, clock effect), and finally the full geometrical edifice of Minkowski space-time.

Einstein's theory of general relativity of 1915 retains all the concepts of special relativity for infinitesimal space-time regions, and supplements them with a new basic ingredient, the properties of free fall. It is these properties which suggest the ideas of the principle of equivalence, the (pseudo-) Riemannian structure of space-time, and finally Einstein's gravitational field equations.

In a recent paper [1], Pirani and the authors have shown that the space-time structure of general relativity theory can be fully explored by observing the free fall of particles and the propagation of light. We give a short summary of these results (which are illustrated in Fig. 1):

* This paper is dedicated to our friend John Archibald Wheeler, geometer and physicist, who celebrated his sixtieth birthday on July 9, 1971.

This work was supported in part by the National Science Foundation (Grant No. GP-34639X). One of the authors (A.S.) did much of this work while visiting the Université Libre de Bruxelles (summer, 1968), Cambridge University (summer, 1970), and the Nordic Institute for Theoretical Atomic Physics (1970-71); he wishes to thank these institutions and Drs. I. Prigogine, D. Sciama, and C. Møller for their kind hospitality. 
Manifold $\boldsymbol{M}$ (coordinate patches, continuity, differentiability)

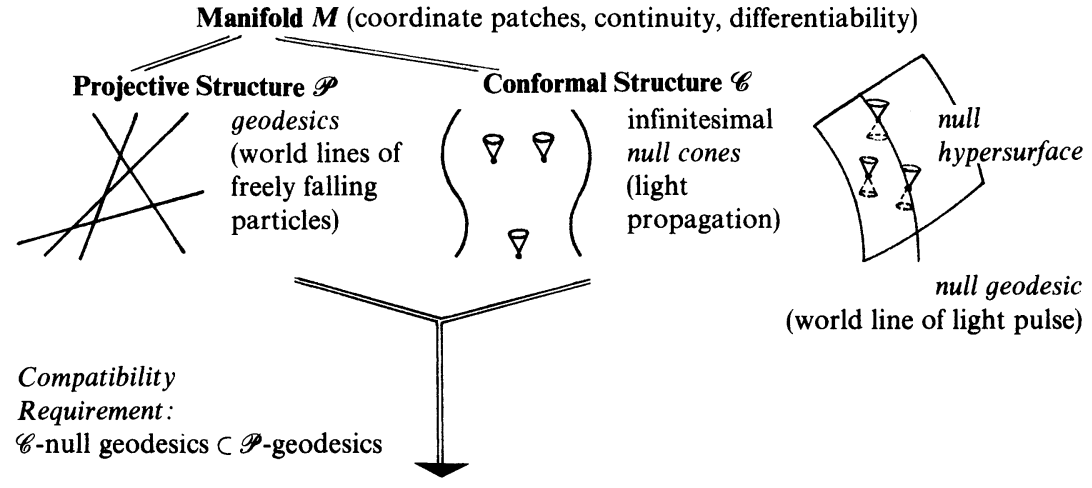

\section{Weyl Structure $\mathscr{W}$}

unique symmetric connection, parallel transport along curves, "length" along null geodesic or along non-null curve $t$ (clock): $V_{A}\|V\| V_{B}$ $\frac{d s_{A}}{\left|V_{A}\right|}=\frac{d s}{|V|}=\frac{d s_{B}}{\left|V_{B}\right|}$

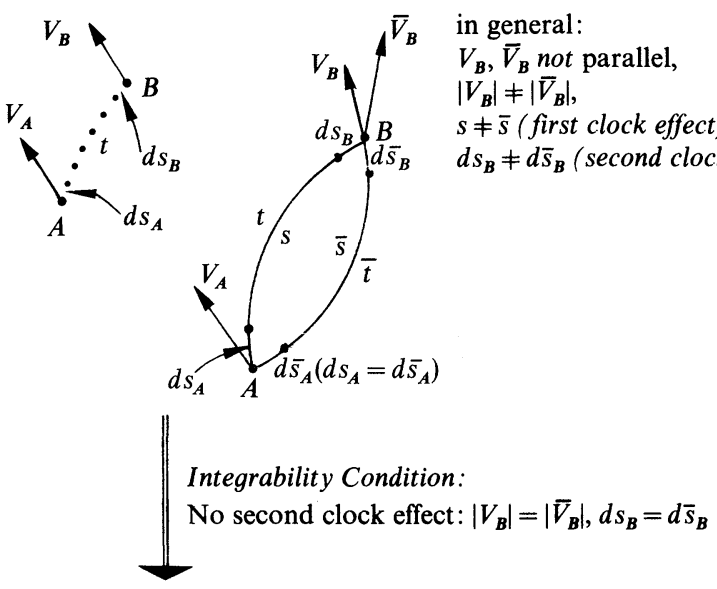

\section{Riemannian Structure $\mathscr{R}$}

unique metric tensor (general relativity theory, gravitational field).

Fig. 1. General scheme of projective, conformal, Weyl and Riemannian structures

The motions of freely falling particles determine the geodesics of space-time (for the present we ignore the fact that not all geodesics are world lines of freely falling particles). A manifold in which some curves with certain properties, the geodesics, have been singled out has a projective structure $\mathscr{P}$. Equivalently, a manifold has a projective structure if it is endowed with a class of symmetric affine connections which all determine the same set of geodesics.

In a projective space, infinitesimal autoparallelism of directions is defined, i.e., the parallel transport of a direction at a point to a neighboring 
point which lies in that same direction; the geodesics are the lines whose tangent directions are autoparallel.

The propagation of light determines at each point of space-time the infinitesimal null cone. A manifold in which a quadratic cone, the null cone, has been singled out in the tangent space of each point has a conformal structure $\mathscr{C}$. Equivalently, a manifold has a conformal structure if it is endowed with a class of (indefinite) metric tensors which are proportional to one another, and thus determine the same null cones.

We shall assume that, in our conformal space, the class of metrics has normal hyperbolic signature. We can then distinguish between timelike, null and space-like directions. The ratio of the lengths of two non-null vectors at the same point is well-defined, and so is the angle between two non-null directions at the same point. Some null curves, the null geodesics, are singled out as follows: A hypersurface which at each of its points is tangent to the null cone is a null hypersurface. A null geodesic is a null curve which is contained in some null hypersurface. We mention in passing that null geodesics can also be characterized geometrically in a conformal space with arbitrary indefinite signature, by adding the requirement that the null curve everywhere point in the direction of the common tangent of the null cone and the null hypersurface.

In a manifold with both projective and conformal structure, a simple and natural compatibility requirement suggests itself, namely that all the null geodesics determined by the conformal structure belong to the class of geodesics determined by the projective structure. Such a manifold with compatible projective and conformal structure has a Weyl [2] structure $\mathscr{W}$.

In a Weyl space, the conformal structure singles out in a natural manner a unique symmetric connection from the class of affine connections which characterize the compatible projective structure. This Weyl connection determines the parallel transport of vectors along a curve, which leaves unchanged the time-like, null or space-like character of a vector, and which leaves constant the ratio of the lengths of two non-null vectors and the angle between them. Geodesics, null or non-null, are completely characterized by the property that their tangential directions are parallelly transported along them. An affine parameter, unique within linear transformations, can be determined along any geodesic. The arc length $s$ can also be defined along any non-null curve and is unique to within linear transformations. This is done by parallelly transporting any non-null vector $V$ along the curve and defining elements of arc $d s$ at different points as equal if they have the same ratio to the magnitude $|V|$ of $V$, i.e., $d s /|V|=$ constant. In a hypothetical Weyl space-time, this construction of arc length along a time-like curve constitutes a clock measuring proper time, which is unique once a zero of time and an initial unit of time have been chosen. 
Consider the parallel transport of a non-null vector $V_{A}$ from a point $A$ to a point $B$ along two different curves $t$ and $\bar{t}$ (lower right-hand diagram in Fig. 1). The resulting vectors at $B, V_{B}$ and $\bar{V}_{B}$, will in general be different: $V_{B} \neq \bar{V}_{B}$. The conformal structure of the Weyl space permits a comparison of the lengths of the two vectors at $B$ and, in general, these will also differ: $\left|V_{B}\right| /\left|\vec{V}_{B}\right| \neq 1$. It is this last property which distinguishes a Weyl space from a Riemannian space. Equivalently, if we construct arc lengths $s$ and $\bar{s}$ along non-null curves $t$ and $\bar{t}$ joining $A$ and $B$, if at $A$ the elements of arc length $d s_{A}$ and $d \bar{s}_{A}$ are equal ${ }^{1}, d s_{A}=d \bar{s}_{A}$, if $d s_{B}=d s_{\underline{A}}$ with respect to the curve $t$ and $d \bar{s}_{B}=d \bar{s}_{A}$ with respect to the curve $t$, then in general $d s_{B} \neq d \bar{s}_{B}$ at $B$. A hypothetical physical space-time which has a Weyl structure, and where the proper time measured by a clock is the Weyl arc length along its' world line, will be called a Weyl space-time. In a Weyl space-time if two clocks, synchronized and identical at an event $A$, are separated and moved along different world lines $t$ and $\bar{t}$ to the same event $B$, then not only will the elapsed times be different, $s \neq \bar{s}$ (the first or usual clock effect of relativity theory), but in general the two clocks will tick at different rates at $B$ (the second clock effect).

Finally, a Weyl space reduces to a Riemannian space $\mathscr{R}$, if and only if a simple integrability condition is satisfied: under parallel transport the magnitude of a vector is always path independent, i.e., $\left|V_{B}\right| /\left|\bar{V}_{B}\right|=1$ in Fig. 1. In space-time, this is equivalent to saying that a Weyl structure reduces to a Riemannian structure if and only if there is no second clock effect.

We can also express the integrability condition in terms of curvature. The unique Weyl connection $\Gamma_{a b}^{c}=\Gamma_{b a}^{c}$ determines a curvature tensor

with symmetries

$$
R_{a b c}^{d}=2 \partial_{[b} \Gamma_{c] a}^{d}-2 \Gamma_{a[b}^{m} \Gamma_{c] m}^{d}
$$

Defining the Ricci tensor

$$
R_{a(b c)}^{d}=R_{[a b c]}^{d}=0 .
$$

$$
R_{a b}=R_{a c b}^{c} \text {, }
$$

the symmetries (1.2) imply

$$
R_{c a b}^{c}=2 R_{[a b]} .
$$

The necessary and sufficient condition for the Weyl structure to reduce to a Riemannian structure is that the Ricci tensor be symmetric:

$$
R_{c a b}^{c} \equiv 2 R_{[a b]}=0 .
$$

We wish to remark that if general relativity theory provides the correct description of nature, then the projective structure of space-time, explored by experiments with freely falling particles, and the conformal

${ }^{1}$ Equal signs here do not mean numerical equality; they refer to comparisons of lengths as discussed above. 
structure, explored by experiments with light propagation, automatically satisfy the compatibility requirement and the integrability condition. Thus the Riemannian space-time structure can be fully explored by observing the world lines of particles in free fall and the propagation of light [2].

It is important and interesting to study geometrical operations and objects which are meaningful in spaces with only conformal structure or with only projective structure, since, as was outlined above, the full structure of a Weyl or Riemannian space can be synthesized from these two.

In a conformal space, Synge [3] used a beautiful "bouncing photon" construction for the Fermi transport of a non-null direction along a non-null curve. The proof was simplified by Pirani [4]. Pirani and Schild [5] gave purely conformal geometrical characterizations of conformal curvature.

In this paper, we give some geometrical constructions in a projective space, in particular the parallel transport along a geodesic of a tangential two-direction and a purely projective characterization of projective curvature. Some of the results reported here were obtained four years ago by the authors and Pirani, who is publishing a somewhat different version of our joint work [6].

Conformal and projective geometrical constructions may give additional insight into the problem of the completion of space-time, Penrose's [7] null infinity, Geroch's [8] treatment of singularities, and Sachs's [9] future time-like infinity.

We wish to thank Felix Pirani for showing us his account of projective curvature prior to publication; our discussion of the extended projective curvature tensor in Section VIII is based on some of his remarks. Also, we wish to thank Paul Sommers for reading our manuscript carefully, and for pointing out a simplification of the assumptions of the theorem in Section IX.

\section{Notation}

Suffixes will be avoided as far as possible. Thus a contravariant vector $A^{a}$ will be denoted by $A$ :

$$
A \leftrightarrow A^{a} .
$$

We shall use " $\wedge$ " to denote the (skew-symmetric) exterior or Grassman product:

$A \wedge B \leftrightarrow 2 A^{[a} B^{b]}=A^{a} B^{b}-B^{a} A^{b}, \quad A \wedge B \wedge C \leftrightarrow 3 ! A^{[a} B^{b} C^{c]}, \quad$ etc. (2.2)

A direction will be denoted by $\lambda A$, where it is understood that this denotes the set of vectors $\{\lambda A \mid \lambda>0\}$. The two-direction spanned by 
vectors $A$ and $B$ will be denoted by $\lambda A \wedge B$, which is understood to denote the set of bivectors $\{\lambda A \wedge B \mid \lambda>0\}$, etc. Our $m$-directions are all oriented.

A covariant vector field $\psi_{a}$ will be characterized by the linear form

$$
\psi(A)=\psi_{a} A^{a},
$$

and a symmetric connection $\Gamma_{a b}^{c}=\Gamma_{b a}^{c}$ by the (coordinate-dependent) bilinear expression

$$
\Gamma(A, B)=\Gamma(B, A) \leftrightarrow \Gamma_{a b}^{c} A^{a} B^{b} .
$$

Consider a curve $t$ with parametrization $\tau$,

$$
t: x=x(\tau) \leftrightarrow x^{a}=x^{a}(\tau) .
$$

The tangent vectors which correspond to this parametrization will be denoted by $T$ :

$$
T=\frac{d x}{d \tau} \leftrightarrow T^{a}=\frac{d x^{a}}{d \tau} .
$$

Conversely, given a smooth vector field $T$, this determines a congruence of curves $t$, which have tangents $T$ and parameters $\tau$ satisfying Eq. (2.6). We shall call the curves of this congruence $T$-curves.

For a vector field $A$ defined along a curve $t$, we denote the (coordinatedependent) ordinary derivative by

$$
\partial_{T} A=\frac{d A}{d \tau} \leftrightarrow \frac{d A^{a}}{d \tau},
$$

and the absolute or covariant derivative with respect to a connection $\Gamma$ by

$$
\nabla_{T} A=\partial_{T} A+\Gamma(A, T) \leftrightarrow \frac{d A^{c}}{d \tau}+\Gamma_{a b}^{c} A^{a} T^{b} .
$$

For a vector field $T$, defined in an $n$-dimensional neighborhood, we also have

$$
\begin{aligned}
& \partial_{T} A=T^{a} \partial_{a} A, \\
& \nabla_{T} A=T^{a} \nabla_{a} A .
\end{aligned}
$$

The Lie bracket of two vector fields $A$ and $B$ is

$$
[A, B]=\partial_{A} B-\partial_{B} A=\nabla_{A} B-\nabla_{B} A .
$$

A curvature tensor $P_{a b c}^{d}$ will be characterized by the vector-valued trilinear form

$$
P(A ; B \wedge C) \leftrightarrow P_{a b c}^{d} A^{a} B^{b} C^{c} .
$$

Throughout this work, it will be understood that all statements are local and that all objects considered satisfy as many smoothness require- 
ments as are convenient. The word "curve" will be used instead of the clumsier "one-dimensional submanifold"; any parametrization of a curve will be assumed to be smooth.

\section{Projective Space; Geodesics}

Two affine connections on a manifold are called projectively equivalent if their geodesics coincide (the affine parametrizations may, of course, differ). This is the case if and only if the corresponding covariant derivatives $\nabla$ and $\nabla^{\prime}$ are related by

$$
\nabla_{A}^{\prime} B=\nabla_{A} B+A \psi(B)+B \psi(A)
$$

or, equivalently, if

$$
\Gamma^{\prime}(A, B)=\Gamma(A, B)+A \psi(B)+B \psi(A),
$$

where $\psi$ is some covariant vector field.

We define a projective structure on a manifold to be an equivalence class of projectively related affine connections. A manifold with a projective structure will be called a projective space. A transformation (3.2) from one affine connection to another (in the same projective structure) will be called a projective transformation.

A one-dimensional projective space is trivial. It is always projectively flat, i.e., it is locally identical with the projective line. In fact, if we permit the possible addition of one single point, then a connected one-dimensional projective space is globally identical to the projective line.

A two-dimensional projective space requires special treatment. For example, its curvature is not measured by the usual projective curvature tensor, which vanishes identically in two dimensions, but by an object of one higher differential order (cf. Section VIII) [2]. We hope to discuss its geometry in a future paper.

In this paper we shall assume (except for Section VIII) that the dimension $n$ of our projective space is greater than two:

$$
n \geqq 3 \text {. }
$$

To study a projective structure, one can either introduce the projective coefficients

$$
\Pi_{a b}^{c}=\Gamma_{a b}^{c}-\frac{2}{n+1} \delta_{(a) d}^{c} \Gamma_{b) d}^{d},
$$

which are invariant under a projective transformation, and have zero trace $\left(\Pi_{a b}^{b}=0\right)$, but which do not constitute a connection, or one can work with a representative connection $\Gamma$ and check the invariance of all 
relevant results under an arbitrary projective transformation (3.2). In our earlier paper [1] we used the first method. Here we find it convenient to use the second one.

Besides the projective transformations (3.2), we shall also wish to consider parameter transformations $\tau^{\prime}=\tau^{\prime}(\tau)$ along a curve $t$, under which the change of the tangent vectors and the covariant derivative are given by

$$
T^{\prime}=\frac{d \tau}{d \tau^{\prime}} T, \quad \nabla_{T^{\prime}}=\frac{d \tau}{d \tau^{\prime}} \nabla_{T} .
$$

The basic geometrical objects in a projective space are the geodesics. They are the curves $t$ which satisfy any of the following equivalent relations: ${ }^{2}$

$$
T \wedge \nabla_{T} T=0 \Leftrightarrow \nabla_{T}(\lambda T)=\mu T \Leftrightarrow \nabla_{T}(v T)=0 .
$$

In space-time the time-like geodesics are the world lines of particles in free fall.

The first Eq. (3.6), $T \wedge \nabla_{T} T=0$, is invariant under both projective transformations and parameter transformations.

The second Eq. (3.6), $\nabla_{T}(\lambda T)=\mu T$, must be interpreted as follows: $\lambda(\tau)$ is an arbitrary positive function along $t$, and $\mu(\tau)$ is a function which is determined by the choice of a particular connection from the projective class of connections, by the choice of the parameter $\tau$, and by the choice of the function $\lambda$. Under a projective transformation (3.2), a parameter transformation (3.5), or a transformation $\lambda(\tau) \rightarrow \lambda^{\prime}(\tau)$, the respective changes in $\mu$ are given by:

$$
\begin{aligned}
& \mu^{\prime}=\mu+2 \lambda \psi(T), \\
& \mu^{\prime}=\left(\frac{d \tau}{d \tau^{\prime}}\right)^{2} \mu+\frac{d^{2} \tau}{d \tau^{\prime 2}} \lambda, \\
& \mu^{\prime}=\frac{\lambda^{\prime}}{\lambda} \mu+\lambda \frac{d}{d \tau}\left(\frac{\lambda^{\prime}}{\lambda}\right) .
\end{aligned}
$$

The third Eq. (3.6), $\nabla_{T}(v T)=0$, must be interpreted as follows: $v(\tau)$ is a function which is determined, uniquely to within linear transformations, by the choice of a particular connection and by the choice of the parameter $\tau$. Under a projective transformation (3.2) or a parameter transformation (3.5), the respective changes in $v$ are given by:

$$
\begin{gathered}
\frac{d}{d \tau}\left(\frac{v^{\prime}}{v}\right)+2 \frac{v^{\prime}}{v} \psi(T)=0, \\
v^{\prime}=\frac{d \tau^{\prime}}{d \tau} v .
\end{gathered}
$$

${ }^{2}$ In this paper, a statement of the form $\alpha=0 \Leftrightarrow \beta=0 \Leftrightarrow \gamma=0$ will always be understood to mean (a) that any one of the three relations imply the other two, and (b) that all three relations hold, i.e., that $\alpha=0, \beta=0, \gamma=0$. 
It is now clear that autoparallellism of a direction is a projective concept, and that geodesics $t$ are completely characterized by the property that their tangent directions $\lambda T$ are autoparallel.

Finally, we make a remark which can often be used to simplify proofs of projective statements (cf. Section X): Given a geodesic $t$ and a smooth parameter $\tau$ on it, there always exist (several) connections $\Gamma^{\prime}$ from the projective class such that $\tau$ is an affine parameter on $t$ with respect to $\Gamma^{\prime}$.

In order to prove this, consider the form

$$
\nabla_{T} T=\mu T
$$

of the equation of the geodesic $t$, where $\mu=\mu(\tau)$. Under a projective transformation, Eq. (3.1), this becomes

$$
\nabla_{T}^{\prime} T=[\mu+2 \psi(T)] T .
$$

If we choose the covariant vector field $\psi$, so that along $t$

$$
\psi(T) \equiv \psi_{a} \frac{d x^{a}}{d \tau}=-\frac{1}{2} \mu,
$$

and this can clearly be done in many ways, then

$$
\nabla_{T}^{\prime} T=0,
$$

so that $\tau$ is an affine parameter along $t$ for the projectively related affine connection $\Gamma^{\prime}(A, B)=\Gamma(A, B)+A \psi(B)+B \psi(A)$.

Equation (3.11) is a weak restriction on the vector field $\psi$. It follows easily that, given two intersecting geodesics $t$ and $s$, with smooth parametrizations $\tau$ and $\sigma$, respectively, then there exist (several) connections $\Gamma^{\prime}$ from the projective class such that, with respect to $\Gamma^{\prime}, \tau$ is affine on $t$ and $\sigma$ is affine on $s$.

\section{The Zig-Zag Construction; a Lemma}

Consider a curve $t$, and direction fields $\lambda X, \lambda Y$ defined along $t$, with the property

$$
X \wedge Y \wedge T=0 \text { along } t .
$$

It is assumed that, all along $t$, any two of the vectors $X, Y, T$ are independent. Our Eq. (4.1a) states that $\lambda X \wedge Y$ is a two-direction which contains the tangential direction $\lambda T ; \lambda X \wedge Y$ is therefore called a tangential two-direction.

We can always, and in many ways, extend $\lambda X$ and $\lambda Y$ smoothly to a neighborhood $\mathcal{N}(t)$ of $t$ such that

$$
X \wedge \nabla_{X} X=0, \quad Y \wedge \nabla_{Y} Y=0 \text { along } t .
$$


These equations state that, at any point of $t$, the $X$-curve and the $Y$-curve through this point are initially geodesic. We shall say that these direction fields on $\mathscr{N}(t)$ are an extension of $\lambda X, \lambda Y$ (on $t$ ) which is initially geodesic.

We can also, and in many ways, extend $\lambda X$ and $\lambda Y$ smoothly to a neighborhood $\mathcal{N}(t)$ of $t$ such that

$$
X \wedge Y \wedge[X, Y]=0 \text { along } t .
$$

This equation states that the $X$-curve through a point of $t$ and the $Y$-curve through a neighboring point of $t$ have a second order intersection, rather than the first order intersection implied by Eq. (4.1a) alone. We shall say that such an extension is strip-forming along $t$.

The Eqs. (4.1) and (4.2) are clearly invariant under projective transformations (3.2). They are also invariant under the changes $X^{\prime}=a X, Y^{\prime}=b Y, T^{\prime}=c T$, where $c$ is an arbitrary function along $t$ and $a$ and $b$ are arbitrary functions in a neighborhood of $t$. Therefore, these equations describe projective relations between the direction fields $\lambda X, \lambda Y, \lambda T$.

We can always select vector representatives $X, Y, T$ of the three direction fields $\lambda X, \lambda Y, \lambda T$ such that

$$
T=X+Y \text { along } t,
$$

where one of the three directions may have to be re-oriented. We then define a vector field $N$ on $t$ by

$$
N=X-Y \text { along } t .
$$

An extension of $\lambda X, \lambda Y$ which is both initially geodesic and stripforming so that all the Eqs. (4.1) to (4.3) are satisfied, may be visualized in terms of the zig-zag or tongs construction of Figure (2), where $\varepsilon$ is an infinitesimal and $A B, B C D, D E F$, etc. (and $A B^{\prime}, B^{\prime} C D^{\prime}, D^{\prime} E F^{\prime}$, etc.) are geodesic arcs.

Lemma. Given a curve $t$, direction fields $\lambda X, \lambda Y$ along $t$ such that $\lambda X \wedge Y$ is a tangential two-direction, and given an extension of $\lambda X, \lambda Y$ which is initially geodesic, i.e., given that Eqs. (4.1) are satisfied, then representative vector fields, characterized by Eqs. (4.3), satisfy the equation

$$
N \wedge \nabla_{T}(X \wedge Y)=X \wedge Y \wedge[X, Y] \text { along } t .
$$

Since the right-hand side of this equation is clearly independent of the choice of connection from the projective class, this must also be true of the left-hand side. A simple calculation confirms this. Thus our lemma is a purely projective statement.

To prove the lemma, we observe that Eq. (4.3a) implies that $\nabla_{T}=\nabla_{X}+\nabla_{Y}$ along $t$, provided the operations on the right-hand side 


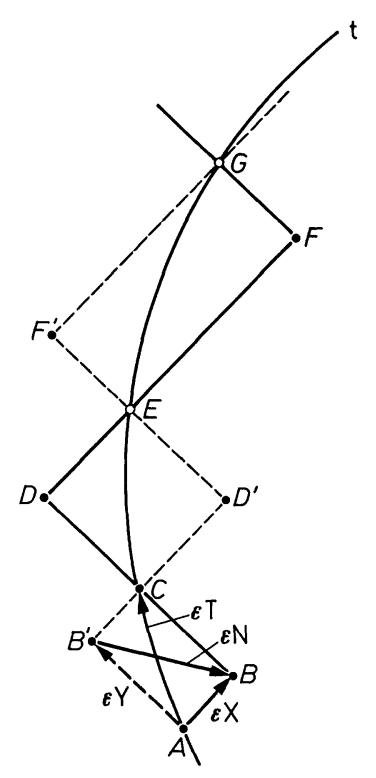

Fig. 2. The Zig-Zag or Tongs construction

are defined. We then find that

$$
\begin{aligned}
X \wedge Y \wedge \nabla_{T} N & =X \wedge Y \wedge\left(\nabla_{X}+\nabla_{Y}\right)(X-Y) \\
& =-Y \wedge\left(X \wedge \nabla_{X} Y\right)-X \wedge\left(Y \wedge \nabla_{Y} Y\right)-X \wedge Y \wedge[X, Y] \\
& =-X \wedge Y \wedge[X, Y] \quad \text { along } t,
\end{aligned}
$$

by Eqs. (4.1b). Since $X \wedge Y \wedge N=0$ along $t$, and therefore

$$
0=\nabla_{T}(X \wedge Y \wedge N)=X \wedge Y \wedge \nabla_{T} N+N \wedge \nabla_{T}(X \wedge Y) \text { along } t
$$

Eq. (4.4) follows and the lemma is proved.

\section{The Parallel Transport along a Geodesic of a Tangential Two-Direction}

Let $t$ be a curve, and let $\lambda X \wedge Y$ be a tangential two-direction along $t$, so that Eq. (4.1a) holds. We shall say that the tangential two-direction is parallel along $t$ if and only if any of the following three equivalent conditions is satisfied:

$\left.\begin{array}{l}X \wedge \nabla_{T}(X \wedge Y)=0 \\ Y \wedge \nabla_{T}(X \wedge Y)=0\end{array}\right\} \Leftrightarrow \nabla_{T}(\lambda X \wedge Y)=\mu X \wedge Y \Leftrightarrow \nabla_{T}(v X \wedge Y)=0$ along $t$. 
The first set of two equations, $X \wedge \nabla_{T}(X \wedge Y)=Y \wedge \nabla_{T}(X \wedge Y)=0$, is invariant under both projective and parameter transformations. The other equivalent equations must be interpreted in an analogous manner to the corresponding equations in (3.6). Thus the parallel transport along a curve of a tangential two-direction is a purely projective concept.

As we shall show in the following two sections, a parallel tangential two-direction exists only for special curves $t$ (geodesics and two-geodesics).

Given a tangential two-direction along a curve $t$, we can always choose independent direction fields $\lambda X, \lambda Y$ along $t$ so that $\lambda X \wedge Y$ is the given tangential two-direction, and we can always choose an extension of $\lambda X, \lambda Y$ which is initially geodesic, i.e., we can always choose $X, Y, T, N$ so that Equations (4.1) and (4.3) are satisfied.

If $t$ is a geodesic,

$$
T \wedge \nabla_{T} T=0,
$$

then Eqs. (5.2) and (4.1a) imply

$$
\begin{aligned}
(X+Y) \wedge \nabla_{T}(X \wedge Y) & =T \wedge \nabla_{T}(X \wedge Y) \\
& =\nabla_{T}(X \wedge Y \wedge T)-X \wedge Y \wedge \nabla_{T} T \\
& =0 \text { along } t .
\end{aligned}
$$

Equation (4.4) of our lemma may be written in the form

$$
(X-Y) \wedge \nabla_{T}(X \wedge Y)=X \wedge Y \wedge[X, Y] \text { along } t,
$$

and hence

$$
X \wedge \nabla_{T}(X \wedge Y)=-Y \wedge \nabla_{T}(X \wedge Y)=\frac{1}{2} X \wedge Y \wedge[X, Y] \text { along } t .
$$

This gives our

First Main Theorem. $A$ tangential two-direction $\lambda X \wedge Y$ along $a$ geodesic $t$ is parallel along $t$ if and only if any initially geodesic extension of $\lambda X, \lambda Y$ is strip-forming along t, i.e., satisfies Eq. (4.2).

This purely projective theorem justifies the zig-zag or tongs construction of Fig. 3a as a geometrical way, by means of geodesics alone, to carry out the parallel transport along a geodesic of a tangential twodirection. The resulting parallel strip is illustrated in Fig. $3 \mathrm{~b}$.

Since the zig-zag or tongs construction only involves geodesics and intersections, it is clear that the relationship between the shaded twodirections at the points $A$ and $B$ in Fig. $3 \mathrm{a}$ is purely projective, i.e., independent of the choice of a particular connection from the projective class. It is not at all obvious that the relationship of the two-directions at $A$ and $B$ is independent of the particular choice of zig-zag or tongs between the two points, i.e., the choice of the vector fields $X, Y, T$. (This is, in fact, true if $t$ is a geodesic, but in general not otherwise.) 

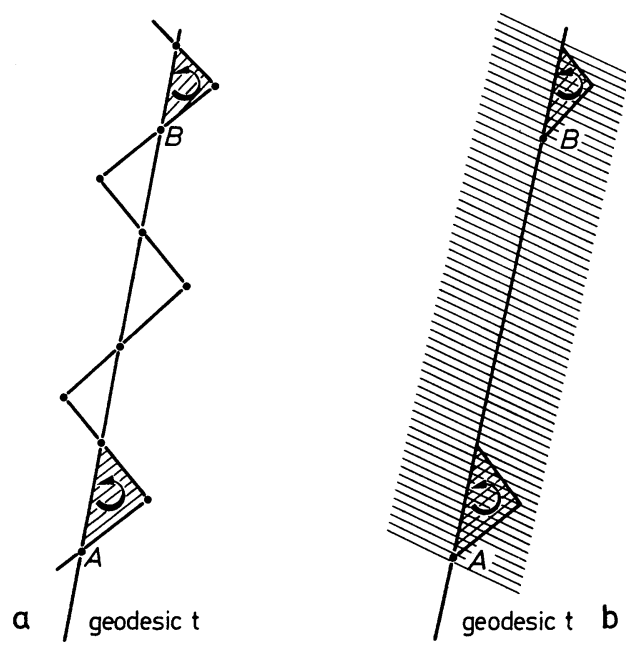

Fig. 3. Zig-Zag construction of parallel transport along a geodesic of a tangential 2-direction

The analytical definitions (5.1) show clearly that the parallel tangential two-direction at $B$ is determined uniquely from an initial tangential two-direction at $A$. It is less obvious (though easy to show) that the relationship of the two-directions at $A$ and $B$ is projective.

The first main theorem permits us to combine the two obvious facts, and makes it clear that the zig-zag construction provides a unique projective parallel transport along a geodesic of a tangential twodirection. Thus a geodesic $t$ and an initial tangential two-direction at one of its points $A$ determines the parallel strip shown in Fig. $3 \mathrm{~b}$, and this strip can be obtained by a zig-zag construction such as the one shown in Fig. 3a.

\section{Generalization of the Concept of Geodesic}

Along a curve $t$, we generalize the concept of tangential direction, one-tangential direction or osculating one-direction

$$
\lambda T,
$$

by defining the two-tangential direction or osculating two-direction by

$$
\lambda T \wedge \nabla_{T} T,
$$

the three-tangential direction or osculating three-direction by

$$
\lambda T \wedge \nabla_{T} T \wedge \nabla_{T}^{2} T,
$$




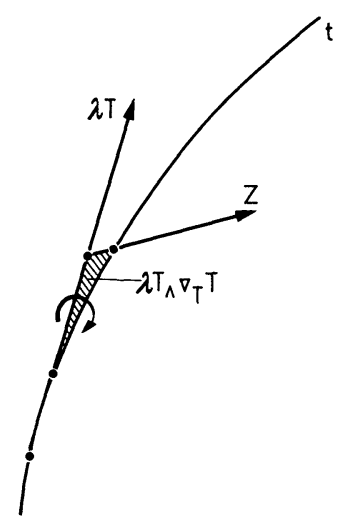

Fig. 4. 1-tangential direction and 2-tangential direction

etc. These, of course, only determine unique $m$-directions if the corresponding $m$-vector does not vanish. We can think of the one-tangential direction as the one-direction which contains two neighboring points of $t$, of the two-tangential direction as the two-direction which contains three neighboring points of $t$, etc. This is shown in Fig. 4.

Remembering that the expressions above are only shorthand for the sets $\{\lambda T \mid \lambda>0\},\left\{\lambda T \wedge \nabla_{T} T \mid \lambda>0\right\}$, etc., it is easy to show that the $m$-tangential direction is a purely projective object, i.e., that the corresponding set of $m$-vectors is independent of the choice of the connection or of the parametrization of $t$.

We generalize the concept of a geodesic or one-geodesic, a curve along which

$$
T \wedge \nabla_{T} T=0,
$$

by defining a two-geodesic as a curve along which

$$
T \wedge \nabla_{T} T \wedge \nabla_{T}^{2} T=0
$$

a three-geodesic as a curve along which

$$
T \wedge \nabla_{T} T \wedge \nabla_{T}^{2} T \wedge \nabla_{T}^{3} T=0,
$$

etc. In a flat projective space, a one-geodesic is a straight line; a twogeodesic is a planar curve or a curve which lies in a two-flat; a threegeodesic is a curve which lies in a three-flat; etc.

Along an arbitrary curve $t$ which is not a one-geodesic, the osculating two-direction $\lambda T \wedge \nabla_{T} T$ is generated by the direction fields $\lambda X, \lambda Y$ where

and therefore

$$
X=\frac{1}{2}\left(T+\nabla_{T} T\right), \quad Y=\frac{1}{2}\left(T-\nabla_{T} T\right) \quad \text { along } t,
$$

$X+Y=T, \quad X-Y=\nabla_{T} T, \quad X \wedge Y=-\frac{1}{2} T \wedge \nabla_{T} T$ along $t . \quad(6.7 \mathrm{~b})$ 
The lemma of Section IV, applied to any initially geodesic extension of $\lambda X, \lambda Y$, gives $\nabla_{T} T \wedge \nabla_{T}(X \wedge Y)=X \wedge Y \wedge[X, Y]$ along $t$, or because of Eq. (6.7b) $\nabla_{T} T \wedge \nabla_{T}\left(T \wedge \nabla_{T} T\right)=-T \wedge \nabla_{T} T \wedge \nabla_{T}^{2} T=-2 X \wedge Y \wedge[X, Y]$ along $t$. This equation together with the identity $T \wedge \nabla_{T}\left(T \wedge \nabla_{T} T\right)=0$ shows:

A non-geodesic curve $t$ is a two-geodesic if and only if any initially geodesic extension of direction fields $\lambda X, \lambda Y$ generating the osculating two-direction $\lambda T \wedge \nabla_{T} T$ is strip forming or, equivalently, if and only if the osculating two-direction is parallelly transported along $t$.

This theorem permits one to test, in terms of a zig-zag construction, whether a non-geodesic curve $t$ is a two-geodesic. It can be generalized to $m$-geodesics.

We now apply the zig-zag construction of Section IV to a curve $t$, which is a two-geodesic but not a one-geodesic. We assume that along $t$ the directions $\lambda X$ and $\lambda Y$ do not lie in the two-tangential direction:

$$
X \wedge T \wedge \nabla_{T} T \neq 0, \quad Y \wedge T \wedge \nabla_{T} T \neq 0 .
$$

We also choose a vector field $Z$ along $t$, so that $\lambda X \wedge Y \wedge Z$ is non-zero and contains the two-tangential direction:

$$
T \wedge X \wedge Y \wedge Z=\nabla_{T} T \wedge X \wedge Y \wedge Z=0 .
$$

This may be done by choosing $Z$ as shown in Fig. 4. We call $\lambda X \wedge Y \wedge Z$ a two-tangential three-direction. Then we have along $t$

$$
\nabla_{T}^{2} T \wedge X \wedge Y \wedge Z=0,
$$

by Eqs. (6.5) and (6.9), and therefore along $t$

$$
\begin{aligned}
T \wedge \nabla_{T}(X \wedge Y \wedge Z) & =\nabla_{T}(T \wedge X \wedge Y \wedge Z)-\nabla_{T} T \wedge X \wedge Y \wedge Z=0, \\
N \wedge \nabla_{T}(X \wedge Y \wedge Z) & =N \wedge \nabla_{T}(X \wedge Y) \wedge Z+N \wedge X \wedge Y \wedge \nabla_{T} Z=0, \\
\nabla_{T} T \wedge \nabla_{T}(X \wedge Y \wedge Z) & =\nabla_{T}\left(\nabla_{T} T \wedge X \wedge Y \wedge Z\right)-\nabla_{T}^{2} T \wedge X \wedge Y \wedge Z=0
\end{aligned}
$$

where we have used Eqs. (6.9) and (6.10) as well as the definition (4.3b) and the lemma (4.4). Since $X, Y, Z$ are independent linear combinations of $T, N$ and $\nabla_{T} T$, we obtain

$$
\left.\begin{array}{l}
X \wedge \nabla_{T}(X \wedge Y \wedge Z)=0 \\
Y \wedge \nabla_{T}(X \wedge Y \wedge Z)=0 \\
Z \wedge \nabla_{T}(X \wedge Y \wedge Z)=0
\end{array}\right\} \Leftrightarrow \nabla_{T}(\lambda X \wedge Y \wedge Z) \quad=\mu X \wedge Y \wedge Z \Leftrightarrow \nabla_{T}(v X \wedge Y \wedge Z)=0
$$

along $t$. This provides a unique projective parallel transport along a twogeodesic of a two-tangential three-direction. The geometrical construction can be visualized as a combination of the zig-zag construction of Fig. 2 and the construction of Fig. 4. 
The results generalize in an obvious fashion to $m$-geodesics. The $m$-tangential direction of an $m$-geodesic $t$ is parallelly transported along $t$. A unique projective parallel transport is defined along an $m$-geodesic of an $m$-tangential $(m+1)$-direction.

\section{Theorems on Projective Parallel Transport}

In Sections III to VI, we have discussed the projective parallel transport of certain $m$-directions along certain curves. We shall now state and prove a series of completeness theorems which show that essentially no other projective parallel transport of $m$-directions is possible:

The First Completeness Theorem. Given an affine connection $\Gamma, a$ curve $t$, and a field of one-directions $\lambda X$ which is parallel along $t$ with respect to $\Gamma$, then the same field of one-directions $\lambda X$ will be parallel along $t$ with respect to every projectively equivalent connection $\Gamma^{\prime}$, if and only if (a) $t$ is a one-geodesic and (b) $\lambda X$ is one-tangential, i.e. $\lambda X=\mu T$.

To prove this theorem, consider the equation

$$
X \wedge \nabla_{T} X=0,
$$

which states that $\lambda X$ is parallel along $t$ with respect to $\Gamma$. Under a projective transformation (3.2), this becomes

$$
X \wedge \nabla_{T}^{\prime} X=X \wedge T \psi(X),
$$

and the right-hand side vanishes for an arbitrary covariant vector field $\psi$ if and only if

$$
X \wedge T=0 \Leftrightarrow \lambda X=\mu T .
$$

This result, together with Eq. (7.1), implies

$$
T \wedge \nabla_{T} T=0 \text {, }
$$

and the theorem follows.

The Second Completeness Theorem. Given an affine connection $\Gamma, a$ curve $t$, and a field of two-directions $\lambda X \wedge Y$ which is parallel along $t$ with respect to $\Gamma$, then the same field of two-directions $\lambda X \wedge Y$ will be parallel along $t$ with respect to every projectively equivalent connection $\Gamma^{\prime}$, if and only if one of the following two alternatives holds:

(A) (a) $t$ is a one-geodesic, and (b) $\lambda X \wedge Y$ is a tangential twodirection which is parallel along t, i.e., $X \wedge Y \wedge T=X \wedge \nabla_{T}(X \wedge Y)$ $=Y \wedge \nabla_{T}(X \wedge Y)=0$, or

(B) (a) $t$ is a two-geodesic, which is not a one-geodesic, and (b) $\lambda X \wedge Y$ is two-tangential, i.e., $\lambda X \wedge Y=\mu T \wedge \nabla_{T} T$. 
We remark that we may assume, without loss of generality, that any two of the vectors $X, Y, T$ are independent. It follows from previous work that the conditions of our theorem are sufficient. To prove that they are necessary, consider the equations

$$
X \wedge \nabla_{T}(X \wedge Y)=0, \quad Y \wedge \nabla_{T}(X \wedge Y)=0,
$$

which state that $\lambda X \wedge Y$ is parallel along $t$ with respect to $\Gamma$. Under a projective transformation (3.2), these become

$$
\begin{aligned}
& X \wedge \nabla_{T}^{\prime}(X \wedge Y)=X \wedge T \wedge Y \psi(X), \\
& Y \wedge \nabla_{T}^{\prime}(X \wedge Y)=Y \wedge X \wedge T \psi(Y),
\end{aligned}
$$

and the two right-hand sides vanish for an arbitrary covariant vector field $\psi$ if and only if, along $t$,

$$
X \wedge Y \wedge T=0 .
$$

This and Eqs. (7.5) imply that $\lambda X \wedge T$ is the tangential two-direction $\lambda X \wedge Y$ and is thus parallel along $t$. Therefore, along $t$,

$$
\begin{aligned}
& X \wedge T \wedge \nabla_{T} T=-T \wedge \nabla_{T}(X \wedge T)=0, \\
& X \wedge T \wedge \nabla_{T} X=-X \wedge \nabla_{T}(X \wedge T)=0 .
\end{aligned}
$$

These equations show that $\nabla_{T} T$ and $\nabla_{T} X$ both lie in the $\lambda X \wedge T$ twodirection, so that

Then

$$
\nabla_{T} X \wedge T \wedge \nabla_{T} T=0 .
$$

$$
X \wedge T \wedge \nabla_{T}^{2} T=\nabla_{T}\left(X \wedge T \wedge \nabla_{T} T\right)-\nabla_{T} X \wedge T \wedge \nabla_{T} T=0,
$$

by Eqs. (7.8) and (7.10). Thus $\nabla_{T}^{2} T$ also lies in the $\lambda X \wedge T$ two-direction, and therefore, along $t$,

$$
T \wedge \nabla_{T} T \wedge \nabla_{T}^{2} T=0 .
$$

If $t$ is a one-geodesic, Eqs. (7.5) and (7.7) show that alternative (A) holds. If $t$ is not a one-geodesic, then Eq. (7.12) shows that $t$ is a twogeodesic, and Eqs. (7.7) and (7.8) imply that $\lambda X \wedge Y$ is two-tangential, so that alternative (B) holds. This completes the proof of our theorem.

It is now clear how the series of theorems continues. We state, without proof,

The General Completeness Theorem. Given an affine connection $\Gamma, a$ curve $t$, and a field of $(m+1)$-directions which is parallel along $t$ with respect to $\Gamma$, then the same field of $(m+1)$-directions will be parallel along $t$ with respect to every projectively equivalent connection $\Gamma^{\prime}$, if and only if one of the following alternatives holds:

(A) (a) $t$ is an m-geodesic, and (b) the $(m+1)$-direction is an $m$-tangential $(m+1)$-direction which is parallel along $t$, or 
(B) (a) $t$ is an $(m+1)$-geodesic, which is not an m-geodesic, and (b) the $(m+1)$-direction is $(m+1)$-tangential.

The only additional parallellisms of $m$-directions along a curve are trivial generalizations of the cases discussed in previous sections and covered by the above completeness theorems. It is sufficient to give one example:

Let $t$ be a two-geodesic which is not one-geodesic, and consider two independent two-tangential three-directions which are projectively parallel along $t$. The two three-directions have the two-tangential direction $\lambda T \wedge \nabla_{T} T$ in common, and therefore they span a two-tangential four-direction. This two-tangential four-direction is parallel along the two-geodesic $t$, and the parallellism is purely projective.

Geometrically, the projective parallel transport of a two-tangential four-direction along $t$ can be carried out by combining the construction of Fig. 4 with two zig-zag constructions as shown in Fig. 2. In detail, this is done as follows: Let $\lambda Z$ be a direction field along the two-geodesic $t$ obtained by the construction of Fig. 4, so that $\lambda T \wedge Z$ is the two-tangential direction $\lambda T \wedge \nabla_{T} T$. Given an initial two-tangential four-direction at a point $A$ of $t$, choose two vector pairs $X, Y$ and $X^{\prime}, Y^{\prime}$ at $A$, such that $X+Y=X^{\prime}+Y^{\prime}=T$ and such that $\lambda X \wedge X^{\prime} \wedge T \wedge Z$ is the initial fourdirection. Perform one zig-zag construction (cf. Fig. 2) along $t$ starting with the vectors $X, Y$ and a second zig-zag construction starting with $X^{\prime}, Y^{\prime}$. This will result in a unique two-tangential four-direction $\lambda X \wedge X^{\prime} \wedge T \wedge Z$ defined along the two-geodesic $t$, and this four-direction is the one obtained by parallel transport along $t$ from the initial fourdirection at $A$.

\section{The Projective Curvature Tensor, Analysis and Algebra}

We start with a brief analytical review of the projective curvature tensor. Any one of the affine connections of the projective class determines a curvature tensor $R$ by means of the Ricci identity which may be written:

$$
R(A ; B \wedge C)=\left(\nabla_{B} \nabla_{C}-\nabla_{C} \nabla_{B}-\nabla_{[B, C]}\right) A .
$$

The explicit expressions for the curvature and Ricci tensors, and for its symmetries are given in Eqs. (1.1)-(1.4).

Under a projective transformation (3.2), the curvature and Ricci tensors transform according to

$$
\begin{aligned}
& R_{a b c}^{\prime d}=R_{a b c}^{d}-2 \delta_{a}^{d} \chi_{[b c]}+2 \chi_{a[b} \delta_{c]}^{d}, \quad \chi_{a b}=\nabla_{b} \psi_{a}-\psi_{a} \psi_{b}, \\
& R_{[a b]}^{\prime}=R_{[a b]}-(n+1) \chi_{[a b]}, \quad R_{(a b)}^{\prime}=R_{(a b)}-(n-1) \chi_{(a b)} .
\end{aligned}
$$


The projective curvature tensor $P$ is a tensor with the same symmetries (1.2) as $R$, but with all traces removed. It is given by

$$
P_{a b c}^{d}=R_{a b c}^{d}-\frac{3}{n+1}\left(\delta_{a}^{d} R_{[b c]}-\delta_{[a}^{d} R_{b c]}\right)-\frac{1}{n-1}\left(\delta_{b}^{d} R_{(a c)}-\delta_{c}^{d} R_{(a b)}\right),
$$

and has the symmetries

which imply $P_{c a b}^{c}=0$.

$$
\begin{aligned}
P_{a(b c)}^{d}=P_{[a b c]}^{d} & =0, \\
P_{a c b}^{c} & =0,
\end{aligned}
$$

It follows from Eqs. (8.2) that $P$ is invariant under a projective transformation, i.e., that $P^{\prime d}{ }_{a b c}=P^{d}{ }_{a b c}$. Weyl [2] has shown that in three or more dimensions, $n \geqq 3, P^{d}{ }_{a b c}=0$ is necessary and sufficient for the flatness of the projective space.

In the following sections we shall give geometrical and physical interpretations for expressions of the form $X \wedge T \wedge P(T ; X \wedge T)$. We shall now discuss algebraic properties of such expressions.

From Eq. (8.3) it follows that, for any vectors $X$ and $T$,

$$
X \wedge T \wedge R(T ; X \wedge T)=X \wedge T \wedge P(T ; X \wedge T) .
$$

We regard $-X \wedge T \wedge P(T ; X \wedge T) \equiv T \wedge P(T ; T \wedge X) \wedge X$ as a trivector valued form, cubic in $T$ and quadratic in $X$ :

$$
-X \wedge T \wedge P(T ; X \wedge T) \equiv Q(T, T, T ; X, X) .
$$

The tensor $Q$ defined by this equation will be called the extended projective curvature tensor. Its components are given by

$$
Q_{p q r s t}^{a b c}=\delta_{(p}^{[a} P_{q r)(s}^{b} \delta_{t)}^{c]},
$$

from which its symmetries can be read off. A somewhat tedious but straightforward calculation shows that

$$
Q_{m a b c n}^{m d n}=\frac{1}{36}\left(n^{2}-1\right) P_{(a b) c}^{d} .
$$

If we combine this with

$$
P_{a b c}^{d}=\frac{2}{3}\left(P_{(a b) c}^{d}-P_{(a c) b}^{d}\right),
$$

which follows from the symmetries (8.4a), we obtain

$$
P_{a b c}^{d}=\frac{48}{n^{2}-1} Q_{m a[b c] n}^{m d n} .
$$

Eqs. (8.7) and (8.10) express each of the tensors $Q$ and $P$ in terms of the other, and thus the two tensors are fully equivalent as measures of projective curvature. 
It follows that the knowledge of $X \wedge T \wedge P(T ; X \wedge T)$ for a sufficiently large set of vector pairs $X, T$ (e.g., for all vectors pairs $X, T$ ) determines the extended projective curvature tensor $Q$, and therefore also fully determines the projective curvature tensor $P$.

We shall conclude this section with a few remarks about the special case of two dimensions,

$$
n=2 .
$$

In a two-dimensional projective space, Eq. (8.7) immediately gives $Q=0$, since $Q$ is completely skew-symmetric in its three contravariant suffixes and since each of these suffixes can only take on one of two values. Eq. (8.10) then shows that

$$
P_{a b c}^{d}=0 .
$$

Thus in two dimensions, projective curvature is not measured by the tensor $P$. Weyl [2] has shown that in two dimensions the tensor

$$
S_{a b c}=S_{[a b] c}=\nabla_{[a} R_{b] c}+2 \nabla_{[a} R_{|c| b]}
$$

is invariant under a projective transformation (3.2), i.e., $S_{a b c}^{\prime}=S_{a b c}$, and that $S=0$ is necessary and sufficient for the flatness of a twodimensional projective space. $S$ has two independent components. We hope to give geometrical and physical interpretations of this tensor in a future paper.

\section{A Projective Analogue of the Equation of Geodesic Deviation}

The qualitative picture of projective curvature is this:

Choose a geodesic $t$, construct a strip of parallel tangential twodirections along $t$, and choose a neighboring geodesic $\bar{t}$ which initially lies in the strip, i.e., $\bar{t}$ goes through a point of the strip and is tangential to the strip at that point. This is shown in Fig. 5.

If the neighboring geodesic $\bar{t}$ always remains in the strip (Fig. 5a), then the space is projectively flat. If the neighboring geodesic $\bar{t}$ curls out of the strip (Fig. 5b), then the space is projectively curved, and the rate at which $\bar{t}$ curls away from the strip measures the projective curvature of the space.

We shall now proceed to describe a construction and to derive a formula, Eq. (9.5) below, which makes these statements more precise.

Construction: Let $t$ be a geodesic with tangent $T$, and $\lambda X \wedge Y$ a tangential two-direction which is parallel along $t$. We select independent vector representatives $X, Y$ such that

$$
\begin{gathered}
T \wedge \nabla_{T} T=0 \text { along } t, \\
T=X+Y \text { along } t .
\end{gathered}
$$



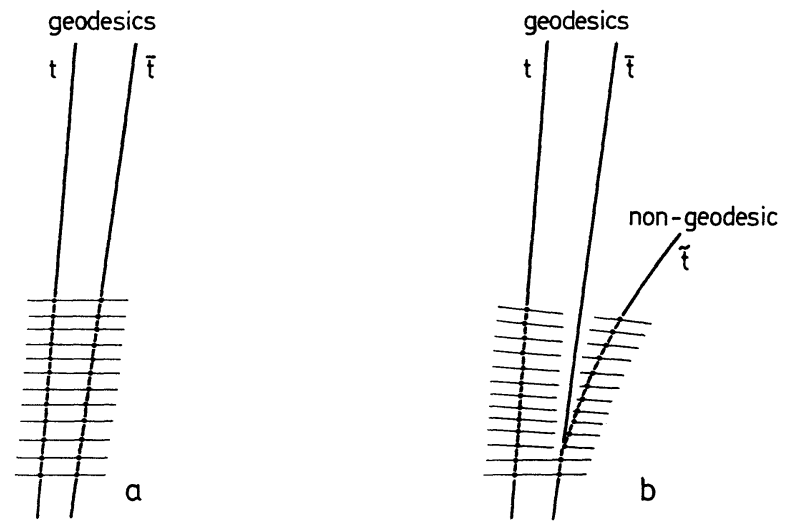

Fig. 5a and b. a Projectively flat space. b Projectively curved space

We now take an initially geodesic extension of the vector fields $X, Y$ to a neighborhood $\mathscr{N}(t)$ of $t$, such that

$$
X \wedge \nabla_{X} X=0, \quad Y \wedge \nabla_{Y} Y=0 \text { along } t .
$$

Then the first main theorem of Section V applies. It tells us that the extension is strip-forming along $t$, i.e., that

$$
X \wedge Y \wedge[X, Y]=0 \text { along } t .
$$

Thus our construction can be carried out geometrically by the zig-zag construction shown in Fig. 3a. Finally, we define vector fields $T$ and $N$ throughout $\mathscr{N}(t)$ by

$$
T=X+Y, \quad N=X-Y \quad \text { in } \mathcal{N}(t) .
$$

This completes our construction.

We can now state our

Second Main Theorem. Given a geodesic $t$ with tangent $T$ and $a$ tangential two-direction $\lambda X \wedge Y$, then vector fields $X, Y, T$ which are obtained by the construction above, i.e., for which Eqs. (9.1) to (9.4) hold, satisfy the equation

$$
X \wedge \nabla_{X}\left(T \wedge \nabla_{T} T\right)=X \wedge T \wedge P(T ; X \wedge T) \text { along } t .
$$

In order to prove this, we observe that along $t$ the following vectors lie in the $\lambda X \wedge Y$ two-direction: $X, Y, T, N, \nabla_{X} X, \nabla_{Y} Y, \nabla_{T} T, \nabla_{T} N$ [by Eqs. (4.5) and (9.3)], hence also $\nabla_{T} X, \nabla_{T} Y, \nabla_{X} Y=\nabla_{T} Y-\nabla_{Y} Y$ and $\nabla_{X} T$ 
$=\nabla_{X} X+\nabla_{X} Y$. Thus the outer product of any three of these vectors is zero along $t$. Therefore

$$
\begin{aligned}
X \wedge \nabla_{X}\left(T \wedge \nabla_{T} T\right) & =X \wedge T \wedge \nabla_{X} \nabla_{T} T \text { along } t, \\
0=\nabla_{T}\left(X \wedge T \wedge \nabla_{X} T\right) & =X \wedge T \wedge \nabla_{T} \nabla_{X} T \text { along } t,
\end{aligned}
$$

and, by subtraction,

$$
X \wedge \nabla_{X}\left(T \wedge \nabla_{T} T\right)=X \wedge T \wedge\left(\nabla_{X} \nabla_{T}-\nabla_{T} \nabla_{X}\right) T \text { along } t .
$$

Equations (9.2a) and (9.3) show that along $t,[X, Y]$ is a linear combination of $X$ and $Y$, and that therefore $\nabla_{[X, Y]} T$ is a linear combination of $\nabla_{X} T$ and $\nabla_{T} T$, so that

$$
X \wedge T \wedge \nabla_{[X, Y]} T=0 \text { along } t .
$$

By Eqs. (8.1) and (8.5), the right-hand side of Eq. (9.7) thus becomes $X \wedge T \wedge P(T ; X \wedge T)$, and Eq. (9.5) follows. This completes the proof of our theorem.

Let us now make some simple remarks:

Clearly the right-hand side $X \wedge T \wedge P(T ; X \wedge T)$ of Eq. (9.5) is invariant under a projective transformation and gets multiplied by $\left(d \tau / \mathrm{d} \tau^{\prime}\right)^{3}$ under a parameter transformation. Therefore the left-hand side $X \wedge \nabla_{X}\left(T \wedge \nabla_{T} T\right)$ must have the same properties, and this can of course be checked by a direct calculation. Thus our second main theorem makes a purely projective statement.

Clearly the tangential three-direction $\lambda X \wedge T \wedge P(T ; X \wedge T)$ depends only on the parallelly transported tangential two-direction $\lambda X \wedge T$ $=\mu X \wedge Y$, and on no other property of the initially geodesic vector field $X$. This is therefore also true of $\lambda X \wedge \nabla_{X}\left(T \wedge \nabla_{T} T\right)$.

Because of our definition (9.4), a $T$-curve $\tilde{t}$ which neighbors the geodesic $t$ remains in the strip of parallel two-directions $\lambda X \wedge Y$, but $\tilde{t}$ is in general not geodesic, i.e., $T \wedge \nabla_{T} T \neq 0$ at points off $t$. The left-hand side of our main Eq. (9.5) gives a projective measure of the rate at which $\tilde{t}$ deviates from a geodesic $\tilde{t}$, which starts from the same initial point in the same initial direction, as shown in Fig. 5b. Equivalently, it measures the rate at which the geodesic $\bar{t}$ curls away from the strip of parallel tangential two-directions. This, roughly, is the geometrical meaning of $X \wedge \nabla_{X}\left(T \wedge \nabla_{T} T\right)$. Our main Eq. (9.5) then shows that this rate of deviation gives information about the projective curvature $P$ of our projective $n$-space.

Our result (9.5) is a close projective analogue of the Jacobi-LeviCivita equation of geodesic deviation in a Riemannian $n$-space [10].

We could use our formula to give a detailed projective construction of $X \wedge T \wedge P(T ; X \wedge T)$, and hence of the curvature $P$ (cf. Section VIII). 


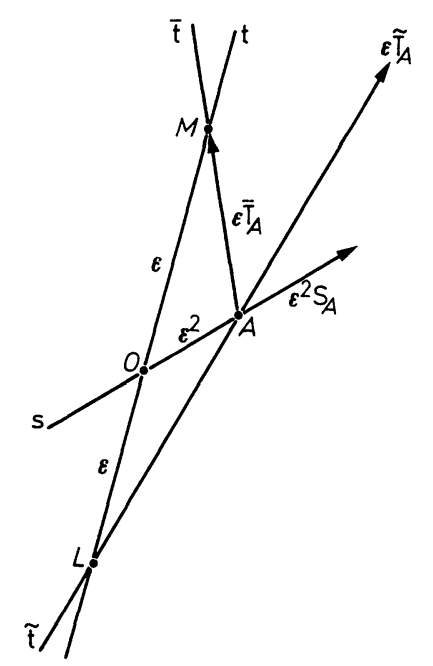

Fig. 6. Construction for the geometrical determination of projective curvature

We prefer, however, to give a simpler geometrical construction of $P$ in the following Section X.

Pirani [6] derives a formula which is essentially equivalent to our Eq. (9.5), and interprets it geometrically.

\section{A Geometrical Construction of Projective Curvature}

We start with the construction shown in Fig. 6 and described in detail by

Construction: Let $t, s$ be two geodesics through $O$ with smooth parameters $\tau, \sigma$ and corresponding tangents $T, S$, such that $\tau(O)=\sigma(O)=0$. Let $L$ and $M$ be points on $t$ with $\tau(L)=-\varepsilon, \tau(M)=\varepsilon$, and let $A$ be a point on $s$ with $\sigma(A)=\varepsilon^{2}$. Join $A$ to $M$ by the geodesic $\bar{t}$, and let $\bar{T}$ be a tangent vector to $\bar{t}$, which depends smoothly on $\varepsilon$, such that $\lim _{\varepsilon \rightarrow 0} \bar{T}_{A}=T_{O}$. Join $L$ to $A$ by the geodesic $\tilde{t}$, and let $\tilde{T}$ be a tangent vector to $\tilde{t}$, which depends smoothly on $\varepsilon$, such that $\lim _{\varepsilon \rightarrow 0} \tilde{T}_{A}=T_{O}$.

The construction is illustrated in Fig. 6 .

With this construction, we state our

\section{Third Main Theorem.}

$$
\lim _{\varepsilon \rightarrow 0} \frac{1}{\varepsilon^{3}} \tilde{T}_{A} \wedge \bar{T}_{A} \wedge S_{A}=\frac{2}{3} S_{O} \wedge T_{O} \wedge P_{o}\left(T_{O}, S_{O} \wedge T_{o}\right) .
$$


We can restate our result in a more intuitive form. Write

$$
\varepsilon \bar{T}_{A}=\overrightarrow{A M}, \quad-\varepsilon \tilde{T}_{A}=\overrightarrow{A L}, \quad-\varepsilon^{2} S_{A}=\overrightarrow{A O} .
$$

We may think of these vectors as infinitesimal connecting vectors between points defined uniquely by the geometric construction. Then we have

$$
\overrightarrow{A L} \wedge \overrightarrow{A M} \wedge \overrightarrow{A O} \approx \frac{2}{3} \overrightarrow{A O} \wedge \overrightarrow{A M} \wedge P_{A}(\overrightarrow{A M} ; \overrightarrow{A O} \wedge \overrightarrow{A M})
$$

with an arbitrarily small error. More precisely, each of the two sides is of order $\varepsilon^{7}$, and the error is of order $\varepsilon^{8}$.

Let us stress that in a projective space the points $A$ and $M$ determine the exact direction of the vector $\overrightarrow{A M}$ at $A$; on the other hand, the length of the vector is less precise and is only determined to the first order in $\varepsilon$. Similar remarks apply to the vectors $\overrightarrow{A O}$ and $\overrightarrow{A L}$ at $A$. However, this slack in the definition of the vectors does not affect the formula (10.3) to the order $\varepsilon^{7}$ which is relevant.

At any point $O$ of our manifold, a sufficiently large number of the purely projective constructions, illustrated in Fig. 6, will determine the extended projective curvature tensor $Q$ and hence, by Eq. (8.10), will fully determine the projective curvature tensor $P$.

In order to prove our main result (10.1), it is convenient to choose a connection $\Gamma$ of the projective class such that both $\tau$ and $\sigma$ are affine parameters with respect to $\Gamma$; this is always possible (cf. Section III). It is also convenient to choose geodesic Fermi coordinates with respect to $\Gamma$, based on $t$, and with origin at $O$. Then

$$
\begin{gathered}
\Gamma=0 \quad \text { along } t, \\
x_{O}=0, \quad x_{M}=\varepsilon T_{O}, \quad x_{L}=-\varepsilon T_{O} .
\end{gathered}
$$

Here and in the following, all equations must be thought of as equations between components of geometrical objects in our special coordinate system. Suffixes are suppressed as far as possible.

Since the geodesic equation

$$
\partial_{S} S=-\Gamma(S, S)
$$

is satisfied along $s$, we have

$$
\begin{aligned}
x_{A} & =\varepsilon^{2} S_{O}+\frac{1}{2} \varepsilon^{4}\left(\partial_{S} S\right)_{O}+O\left(\varepsilon^{6}\right) \\
& =\varepsilon^{2} S_{O}-\frac{1}{2} \varepsilon^{4} \Gamma_{0}\left(S_{O}, S_{O}\right)+O\left(\varepsilon^{6}\right),
\end{aligned}
$$

and thus, by Eq. (10.4),

$$
x_{A}=\varepsilon^{2} S_{O}+O\left(\varepsilon^{6}\right)
$$


We also have

$$
\begin{aligned}
S_{A} & =S_{O}+\varepsilon^{2}\left(\partial_{S} S\right)_{O}+O\left(\varepsilon^{4}\right)=S_{O}+O\left(\varepsilon^{4}\right), \\
\left(\Gamma_{a b}^{d}\right)_{A} & =\varepsilon^{2}\left(\partial_{c} \Gamma_{a b}^{d}\right)_{O} S_{O}^{c}+O\left(\varepsilon^{4}\right), \\
\left(\partial_{c} \Gamma_{a b}^{d}\right)_{A} & =\left(\partial_{c} \Gamma_{a b}^{d}\right)_{O}+O\left(\varepsilon^{2}\right), \\
\left(\partial_{e} \partial_{c} \Gamma_{a b}^{d}\right)_{A} & =\left(\partial_{e} \partial_{c} \Gamma_{a b}^{d}\right)_{O}+O\left(\varepsilon^{2}\right) .
\end{aligned}
$$

Along the geodesic $\bar{t}$, let $\bar{\tau}$ be an affine parameter with respect to $\Gamma$, such that $\bar{\tau}(A)=0$ and $\bar{\tau}(M)=\varepsilon$, and let $\bar{T}$ be the corresponding tangent vector. Then

$$
\begin{aligned}
& \partial_{\bar{T}} \bar{T}^{d}=-\Gamma_{a b}^{d} \bar{T}^{a} \bar{T}^{b}, \\
& \partial_{\bar{T}}^{2} \bar{T}^{d}=-\left(\partial_{c} \Gamma_{a b}^{d}\right) \bar{T}^{a} \bar{T}^{b} \bar{T}^{c}+2 \Gamma_{a m}^{d} \Gamma_{b c}^{m} \bar{T}^{a} \bar{T}^{b} \bar{T}^{c}, \\
& \partial_{\bar{T}}^{\frac{3}{T^{d}}}=-\left(\partial_{e} \partial_{c} \Gamma_{a b}^{d}\right) \bar{T}^{a} \bar{T}^{b} \bar{T}^{c} \bar{T}^{e}+\cdots,
\end{aligned}
$$

where the dots indicate terms, each of which contains an undifferentiated $\Gamma$ as a factor.

Since $X_{M}$ is on $\bar{t}$ and $\bar{\tau}(M)=\varepsilon$, we have, by expansion about $A$,

$$
\begin{aligned}
X_{M}= & X_{A}+\varepsilon \bar{T}_{A}+\frac{1}{2} \varepsilon^{2}\left(\partial_{\bar{T}} \bar{T}\right)_{A}+\frac{1}{6} \varepsilon^{3}\left(\partial_{\bar{T}}^{2} \bar{T}\right)_{A} \\
& +\frac{1}{24} \varepsilon^{4}\left(\partial_{\bar{T}}^{3} \bar{T}\right)_{A}+O\left(\varepsilon^{5}\right) .
\end{aligned}
$$

Using Eqs. (10.10) and (10.9), and the expressions (10.5) and (10.7) for $X_{M}$ and $X_{A}$, this becomes, on division by $\varepsilon$,

$$
\begin{aligned}
T_{O}^{d}= & \bar{T}_{A}^{d}+\varepsilon S_{O}^{d}-\frac{1}{6} \varepsilon^{2}\left(\partial_{c} \Gamma_{a b}^{d}\right)_{O} \bar{T}_{A}^{a} \bar{T}_{A}^{b} \bar{T}_{A}^{c}-\frac{1}{2} \varepsilon^{3}\left(\partial_{c} \Gamma_{a b}^{d}\right)_{O} \bar{T}_{A}^{a} \bar{T}_{A}^{b} S_{O}^{c} \\
& -\frac{1}{24} \varepsilon^{3}\left(\partial_{e} \partial_{c} \Gamma_{a b}^{d}\right)_{O} \bar{T}_{A}^{a} \bar{T}_{A}^{b} \bar{T}_{A}^{c} \bar{T}_{A}^{e}+O\left(\varepsilon^{4}\right) .
\end{aligned}
$$

It follows that $\bar{T}_{A}=T_{O}-\varepsilon S_{O}+O\left(\varepsilon^{2}\right)$, and substituting this into the terms of order $\varepsilon^{2}$ and $\varepsilon^{3}$ of Eq. (10.12), we obtain

$$
\bar{T}_{A}=T_{O}-\varepsilon S_{O}+\frac{1}{3} \varepsilon^{3} R_{O}\left(T_{O} ; S_{O} \wedge T_{O}\right)+O\left(\varepsilon^{4}\right) .
$$

In deriving this equation, we have used the facts that

$$
\left(T^{c} \partial_{c} \Gamma\right)_{o}=\left(T^{e} T^{c} \partial_{e} \partial_{c} \Gamma\right)_{O}=0 \quad \text { and } \quad\left(R_{a b c}^{d}\right)_{O}=2\left(\partial_{[b} \Gamma_{c] a}^{d}\right)_{O},
$$

which follow from Eq. (10.4).

A similar expression for $\tilde{T}_{A}$ is obtained by systematically changing $\bar{t}, \bar{\tau}, \bar{T}, M, \varepsilon$ into $\tilde{t}, \tilde{\tau}, \tilde{T}, L,-\varepsilon$ in the above derivation. It is

$$
\tilde{T}_{A}=T_{O}+\varepsilon S_{O}-\frac{1}{3} \varepsilon^{3} R_{O}\left(T_{O} ; S_{O} \wedge T_{O}\right)+O\left(\varepsilon^{4}\right) .
$$

Forming the outer product of $\tilde{T}_{A}, \bar{T}_{A}$ and $S_{A}$ which are given, respectively, by Eqs. (10.14), (10.13) and (10.8), we obtain

$$
\begin{aligned}
\tilde{T}_{A} \wedge \bar{T}_{A} \wedge S_{A} & =\frac{2}{3} \varepsilon^{3} S_{0} \wedge T_{O} \wedge R_{O}\left(T_{O} ; S_{O} \wedge T_{O}\right)+O\left(\varepsilon^{4}\right) \\
& =\frac{2}{3} \varepsilon^{3} S_{O} \wedge T_{O} \wedge P_{O}\left(T_{O} ; S_{O} \wedge T_{O}\right)+O\left(\varepsilon^{4}\right) .
\end{aligned}
$$


It is clear that this formula remains unchanged if we replace $\bar{T}_{A}, \tilde{T}_{A}$ by any other tangent vectors at $A$ to the geodesics $\bar{t}$ and $\tilde{t}$, which depend smoothly on $\varepsilon$ and which tend to $T_{o}$ as $\varepsilon \rightarrow 0$. Equation (10.1) now follows, and this completes the proof of our theorem.

We remark that we can extract from our proof and Eqs. (10.13), (10.14), and (10.8) the following

Affine Theorem. In an affine space, let $t, s$ be two geodesics through $O$ with affine parameters $\tau, \sigma$ and corresponding tangents $T, S$, such that $\tau(O)=\sigma(O)=0$. Let $L$ and $M$ be points on $t$ with $\tau(L)=-\varepsilon, \tau(M)=\varepsilon$, and let $A$ be a point on $s$ with $\sigma(A)=\varepsilon^{2}$. Let $\bar{t}$ be the geodesic joining $A$ to $M$, and let $\bar{T}$ be its tangent vector with respect to the affine parameter $\bar{\tau}$ which satisfies $\bar{\tau}(A)=0$ and $\bar{\tau}(M)=\varepsilon$. Similarly, let $\bar{t}$ be the geodesic joining $L$ to $A$, and let $\tilde{T}$ be its tangent vector with respect to the affine parameter $\tilde{\tau}$ which satisfies $\tilde{\tau}(A)=0$ and $\tilde{\tau}(L)=-\varepsilon$. Then

$$
\lim _{\varepsilon \rightarrow 0} \frac{1}{\varepsilon^{3}}\left(\bar{T}_{A}-\tilde{T}_{A}+2 \varepsilon S_{A}\right)=\frac{2}{3} R_{O}\left(T_{O} ; S_{O} \wedge T_{O}\right) .
$$

With the definitions (10.2), which now determine the vectors $\overrightarrow{A M}, \overrightarrow{A L}, \overrightarrow{A O}$ precisely, this may be rewritten in the form

$$
\overrightarrow{A M}+\overrightarrow{A L}-2 \overrightarrow{A O} \approx-\frac{2}{3} R_{A}(\overrightarrow{A M} ; \overrightarrow{A O} \wedge \overrightarrow{A M}),
$$

where each of the two sides is of order $\varepsilon^{4}$ and the error is of order $\varepsilon^{5}$.

\section{Space-Time Geometry of Free Fall}

The term "space-time geometry of free fall" will mean the following:

In the (pseudo-) Riemannian space-time of general relativity theory, or in a hypothetical Weyl space-time, we consider the motion (geometrically, the world lines) of particles in free fall. The particles are not supposed to carry physical clocks, nor do we suppose that we have (infinitesimal) rigid rods available. The motions of particles in free fall give a physical realization of some of the geodesics of space-time, namely of those geodesics which are time-like with respect to the conformal structure of space-time. No use is made of the conformal structure, besides the fact that the world line of a particle is automatically time-like, and the theoretical fact that for any Riemannian or Weyl structure the timelike directions at an event are very numerous and, in fact, fill the interior of some four-dimensional cone.

It is clear that all the constructions of Sections IV to X and of Figs. 2-6 can be carried out with curves and vectors which are all time-like. These projective geometrical constructions can then be reinterpreted in terms of idealized physical experiments, each of which involves the 


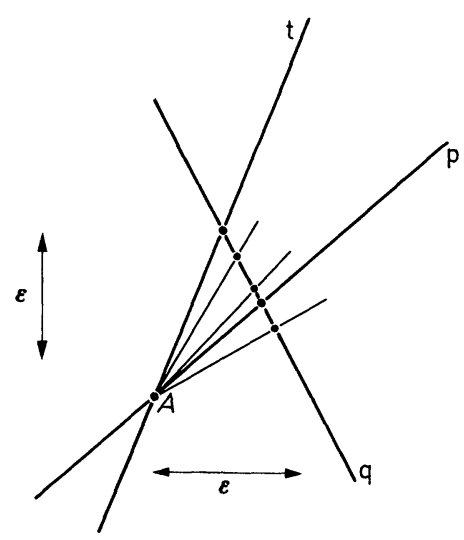

Fig. 7. Tangential 2-direction to $t$ at $A$, which contains $p$

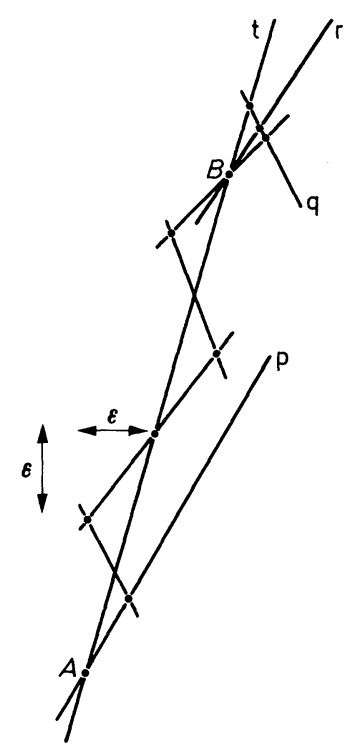

Fig. 8. Parallel transport along a particle $t$ of a tangential 2-direction

motion of particles, observations of a particle moving through a series of events, and of several particles meeting at one event.

All lines shown in the following figures are the world lines of particles in free fall, i.e., time-like geodesics. We shall simply call them particles.

Consider a particle $t$, and a particle $p$ through an event $A$ on $t$, as shown in Fig. 7. Let $q$ be a particle which meets $p$ and $t$ in events near $A$. The set of all particles through $A$ which meet such a $q$ on the same side of $t$ will be called the tangential two-direction to $t$ at $A$ which contains $p$. Geometrically, the two-direction is the set of all time-like directions at $A$ which are coplanar with $t$ and $p$ and lie on the same side of $t$. The last requirement provides a tangential two-direction with an orientation. Physically, we may say that the two-direction consists of the initial elements of the motions of all particles which are shot out from $t$ at event $A$ in the same spatial direction with different (physically possible) velocities.

Let $A$ and $B$ be two events on particle $t$. We say that the tangential two-direction to $t$ at $A$ which contains a particle $p$ and the tangential two-direction at $B$ which contains a particle $r$ are parallel with respect to $t$ if the infinitesimal zig-zag construction shown in Fig. 8 is possible. Given $t, A, B, p$, all particles $r$ through $B$ for which such a zig-zag construction exists will automatically lie in the same tangential twodirection at $B$. 
The constructions or physical experiments illustrated in Figs. 7 and 8 involve the limiting process $\varepsilon \rightarrow 0$. We may think in terms of actual limiting sequences of physical experiments. Or we may adopt the physicist's point of view, think of $\varepsilon$ as a finite and small number, and take it for granted that all our statements are approximate with a small and finite error.

Finally, we may consider the construction of Fig. 6 to be a physical experiment by restricting $t$ and $s$, and hence $\bar{t}, \tilde{t}$ for small $\varepsilon$, to be time-like geodesics, so that they are particles. Then Eq. (10.1) determines $S \wedge T \wedge P(T ; S \wedge T)$ for time-like vectors $T$ and $S$.

It is obvious that time-like vectors are sufficiently numerous, so that, at any event $O$, a sufficiently large number of such experiments will completely determine the extended projective curvature tensor $Q$. Hence, by Eq. (8.10), the projective curvature tensor $P$ of a Riemannian or hypothetical Weyl space-time can be fully explored by free fall experiments.

\section{References}

1. Ehlers, J., Pirani,F.A.E., Schild,A.: General relativity, papers in honour of J. L. Synge (ed. L. O'Raifeartaigh) 63-84. Oxford: Clarendon Press 1972. See also N. M. J. Woodhouse, J. Math. Phys. 14, 495-501 (1973).

2. Weyl,H.: Raum, Zeit, Materie, Fifth edition, Berlin: Springer 1923, Nachr. Ges. Wiss. Göttingen (1921), 99-112; K. L. Stellmacher, Math. Ann. 123, 34-52 (1951).

3. Synge, J. L.: Relativity: the general theory Chap. III, § 8. Amsterdam: North-Holland 1960.

4. Pirani,F.A.E.: Bull. Acad. Polonica, Math.-Astr.-Phys. Series, 13, 239-242 (1965).

5. Pirani, F. A.E., Schild, A.: Bull. Acad. Polonica, Math.-Astr.-Phys. Series, 9, 543-547 (1961); Perspectives in geometry and relativity, essays in honor of Václav Hlavatý (ed. B. Hoffmann) 291-309. Bloomington: Indiana University Press 1966.

6. Pirani,F.A.E.: Symposia Mathematica, Rome (to be published).

7. Penrose, R.: Battelle Rencontres 1967, Lectures in Mathematics and Physics (ed. C. M. DeWitt and J. A. Wheeler), 121-235. New York: Benjamin 1968.

8. Geroch, R.: Ph. D. thesis, Princeton University (1967); Ann. Physics 48, 526-540 (1968); J. Math. Phys. 9, 450-465 (1968).

9. Eardly, D., Sachs, R. K.: J. Math. Phys. 14, 209-212 (1973). See also D. Eardley, Ph.D. Thesis, University of California at Berkeley (1972).

10. Levi-Civita,T.: The absolute differential calculus, Chap. VII, 208-220; London: Blackie 1927, J. L. Synge and A. Schild, Tensor Calculus, Chap. 3, Sec. 3.3. University of Toronto Press 1949.

\section{J. Ehlers}

MPI f. Physik und Astrophysik

D-8000 München 40

Föhringer Ring 6

Federal Republic of Germany

\section{A. Schild}

The University of Texas

Department of Physics

Austin, Texas 78712 USA 